\title{
International expertise on education programs and Neuronet inititaive
}

\author{
Y.L. Orlov ${ }^{1,2 *}$, A.N. Savostyanov ${ }^{1,2,3}$, T.G. Amstislavskaya ${ }^{2,3}$, L.I. Aftanas ${ }^{2,3}$ \\ ${ }^{1}$ Institute of Cytology and Genetics SB RAS, Novosibirsk, Russia \\ ${ }^{2}$ Novosibirsk State University, Novosibirsk, Russia \\ ${ }^{3}$ Scientific Research Institute of Physiology and Basic Medicine, Novosibirsk, Russia \\ *e-mail:orlov@bionet.nsc.ru
}

Key words: bioinformatics, neurobiology, education, Neuronet initiative, international science exchanges

National technological initiatives assume development of novel educational programs on modern perspective technologies - neurobiology, neuroinformatics, bioinformatics, analysis of big data in genomics. The BGRS (Bioinformatics of Genome Regulation and Structure) biannual conference series in Novosibirsk became a kernel of international cooperation and student exchange programs, presentation of novel software in interdisciplinary fields of biology, informatics and Life Sciences overall.

National "Neuronet" initiative in Russia [1, 2] presents novel type of strategic science and commercial projects establishing leading positions in world research. Several regional Neuronet-centers in the format of non-commercial partnerships will be created in Russia. Among them, the Pacific Center in partnership with the Far Eastern Federal University, the Volga Region Center in partnership with the Samara State Medical University and the company "BiTronics", the Novosibirsk Center in partnership with the Research Institute of Physiology and Basic Medicine of SB RAS, as well as the Neuronet-center "St. Petersburg" in partnership with the company "MDG-Innovation". In addition, the centers will be created in partnership with the Moscow Institute of Physics and Technology (MIPT) and the Skolkovo Institute of Science and Technology (SkTech). The Center in St. Petersburg will specialize in projects related to artificial intelligence. Far Eastern University is interested in developing and promoting technologies of augmented and virtual reality. Specific interest of MIPT Center will also be artificial intelligence, and the Novosibirsk Neuronet-Center will focus on pharmaceuticals and medicine.

The international seminar on the discussion of scientific perspectives in the field of neurotechnologies will be held in the frames First Russian-Chinese Workshop on Bioinformatics and System Biology in the form of a round table with open discussion. It will present the development of methodological recommendations for the selection and involvement of the expert community of the scientific and technical sphere for the discussion and evaluation of roadmap projects of the National Technological Initiative in Russia. Foreign experts will share their experience in international education programs on bioinformatics and National education initiatives.

The authors will present their vision of the existing projects of road maps of the National Technological Initiative in the field of neurotechnologies, fundamental medicine and bioinformatics in the format of open discussion.

Russian and international experts in the field of bioinformatics and neurobiology will include several science groups from Europe and Asia.

Three delegations from largest Chinese Universities (Shanghai Jiao Tong University, Zhejiang University and Huazhong Agricultural University) will take part in the scientific seminar: Prof. Xiaodong Zhao, Prof Ming Chen, Dr Yan Li, Prof. Hong-Yu Zhang. The 
neurobiology experts from Academia Sinica, Taiwan, will share their expertise - Profs Arthur C. Tsai, Hung-Ming Chang, Ya-Ling Yang.

The European group will be presented by Spain, the Netherlands, Italy, Germany, Finland, Austria and Norway. The participants are: Dr Rubén V. Rial, University of the Balearic Islands, Palma de Mallorca; Prof. Hans V. Westerhoff, and Dr. Matteo Barberis, University of Amsterdam, Amsterdam, Holland; Prof. Gianluca Tell, University of Udine, Italy; Dr. Olga Krebs, Heidelberg Institute for Theoretical Research, Heidelberg, Germany; Prof. Juha Kantanen, Institute of Natural Resources of Finland, Jokiainen, Finland; Dr. Fyodor Kondrashov, Institute of Science and Technology, Klosterneuburg, Austria; Dr. Maxim Zakharsev, Norwegian University of Natural Sciences, Oslo.

The invited speakers - medical doctors and bioinformaticians from Russia will continue the discussion about national programs in neurobiology: Irina M. Larina, Institute of Mathematical Problems of Biology RAS (IMPB RAS), Alexey A. Lagunin, Russian National Research Medical University named after N.I.Pirogov, Moscow; Maria G. Samsonova, Saint-Petersburg Polytechnic University, Saint-Petersburg.

The seminar presentations on national international education programs will be started by Prof. RalfHofestädt, Bielefeld University, Germany. He will discuss German-Chinese network on bioinformatics and education in bioinformatics. Dr. Marko Đorđević, Belgrade University, Serbia, will tell about Integrating computational systems biology and bioinformatics in research and education. Drs. Tatiana Tatarinova and Cecile Ben (University of La Verne, USA, and University of Toulouse, France) will give educational program review "Bioinformatics: science of a toolbox?"

Bioinformatics, as an interdisciplinary field, is a case example for analysis of science history perspectives. Thus, Prof. M.Chen, Zhejiang University, Hangzhou, China will present professional initiatives in bioinformatics in China. There are more than 30 bioinformatics related societies/organizations are set up to promote the bioinformatics development. Scientists from European countries are attracted to work in China by the improved research and funding environment. Thus, International cooperation research institutes/centers are established, e.g. the Max Planck-Chinese Academy of Science Partners Institute in Computational Biology in Shanghai, which employs a number of European scientists and plays key roles in facilitating international collaborations [3].

During the seminar the analytical materials on increasing the effectiveness of the implementation of the activities of the roadmap projects of the National Technological Initiative will be discussed. We aim develop methodological recommendations, including on the use of educational process starting from the example of the Department of Medicine and Psychology and the Humanitarian Institute of Novosibirsk State University.

Acknowledgements: The participation of the incited speakers at the BGRS conference and the workshop has been supported by Russian Ministry of Science project 28.12487.2018/12.1.

\section{References}

1. http://nti2035.ru/markets/neuronet

2. http://rusneuro.net/

3. Chen M., Harrison A., Shanahan H., Orlov Y. (2017 ) Biological Big Bytes: Integrative Analysis of Large Biological Datasets. J. Integrative Bioinformatics. 14(3). doi: 10.1515/jib-2017-0052. 